\title{
Hypoxic Ischemic Encephalopathy and Therapeutic Hypothermia
}

\author{
James A. Gill, BSN, RNC-NIC* \\ Regis University, Jesuit, Catholic University in Colorado, USA
}

\begin{abstract}
The aim of this paper is to explore the diagnosis known as Hypoxic Ischemic Encephalopathy (HIE) and an intervention called body cooling or therapeutic hypothermia. This literature review will relate the diagnosis to the clinical setting and investigate the implications for the neonatal nurse practitioner (NNP). This research paper will define the diagnosis and discuss the incidence, etiology, pathophysiology, diagnosis, management, and implications for the NNP. Discussion of HIE is warranted and relevant to all neonatal intensive care unit (NICU) employees because nurses, respiratory therapists, nurse practitioners, and neonatologists are likely to encounter HIE in clinical practice. Knowledge of the disease process and this treatment modality is crucial when caring for patients. Although HIE treatment has multiple approaches, this paper will focus on therapeutic hypothermia as the intervention. The research cited throughout the paper is reputable and founded in evidence-based practice.
\end{abstract}

\section{Keywords}

Hypoxic Ischemic Encephalopathy (HIE), Body cooling, Therapeutic hypothermia, Neonate

\section{Introduction, Risk Factors, and Incidence}

Hypoxic ischemic encephalopathy (HIE) is a brain injury caused by inadequate cerebral blood flow, which occurs secondary to a hypoxic ischemic event during the prenatal, intrapartum, or postpartum time periods. Prime examples of hypoxic ischemic injuries can be divided into two categories: maternal risk factors and perinatal risk factors. Maternal risk factors include thromboembolic disorders, hypertension, infection, placental abnormalities, and illicit drug use. Perinatal risk factors include placental abruption, uterine rupture, cord prolapse, placenta previa, shoulder dystocia, etc. Severity ranges from mild with a full recovery to severe with long-term adverse outcomes. This is a serious complication of full term birth that affects approximately 1.5 to 2.5 neonates per one thousand live births [1]. In fact, an estimated $60 \%$ of infants affected by HIE will experience disabilities such as epilepsy, mental impairments, and cerebral palsy. Therapeutic hypothermia or body cooling, which decreases the metabolic demands of the body and minimizes brain sequelae, is a treatment option for neonates who meet specific inclusion criteria.

\section{Presentation}

Clinical manifestations of HIE typically present within the postpartum period. Manifestations include abnormal heart rate tracings, abnormal arterial blood gas results, low APGAR scores, meconium stained amniotic fluid, decreased tone, depressed reflexes, seizure activity, and resuscitation necessitating the need for respiratory support [1]. The hypoxic ischemic event can cause hypoxemia, hypercapnia, electrolyte disturbances, and metabolic acidosis, which ultimately lead to encephalopathy and organ dysfunction that negatively impacts liver and renal function [2]. Obstruction of cerebral blood flow is the primary cause of the events associated with HIE, which can lead to progressive injury. Brain damage associated with HIE occurs either in the cortical region or in deeper brain structures such as the basal ganglia and/or thalamus. Depending on the degree of brain damage, the neonate can present with hearing loss, learning disabilities, motor dysfunction, cerebral palsy, and death [2]. The pathophysiologic results of hypoxic ischemic injuries are complicated; however, medical professionals now better understand the disease process.

Clinical presentation can be described as mild, moderate, or severe according to the Sarnat scale that was created in 1976. Sarnat Stage I describes mild HIE in a neonate who is

*Corresponding author: James A Gill, BSN, RNC-NIC, Regis University, Jesuit, Catholic University in Colorado, 4561 Range Creek Drive Colorado Springs, CO 80922, USA, Tel: (719)-6944075

Accepted: January 29, 2022

Published online: January 31, 2022

Citation: Gill JA (2022) Hypoxic Ischemic Encephalopathy and Therapeutic Hypothermia. Clin Pediatr Res 6(1):99-102 
hyperalert with normal activity, normal or slightly elevated muscle tone, a weak suck, a strong moro reflex, dilated pupils, tachycardia, and a normal respiratory rate [3]. Alternatively, Sarnat Stage II (moderate HIE) presents with lethargy, decreased activity, mild hypotonia, a weak or absent suck, a weak moro reflex, constricted pupils, and periodic breathing [3]. Finally, Sarnat Stage III (severe HIE) includes manifestations such as stupor/coma, flaccid posture, decerebrate posturing, absent suck and moro reflexes, variable pupil size and heart rate, and apnea [3]. This exam is important when determining the degree of HIE and when deciding upon the best course of action. It is important to note that HIE is a progressive diagnosis; therefore it is crucial that medical professionals repeat the Sarnat exam several times because a patient who initially presents with mild characteristics can develop more severe encephalopathy over time [3].

\section{Diagnosis}

An HIE diagnosis is multifactorial, and it is based upon a combination of maternal/perinatal history, thorough physical and neurological assessments, laboratory results, and imaging [2]. The healthcare team must rule out potential diagnoses that have similar manifestations such as inborn errors of metabolism, infection, stroke, genetic anomalies, subgaleal hemorrhage, and congenital neuromuscular disorders [2]. Laboratory testing includes a complete blood count (CBC) to assess for infection and/or thrombocytopenia, blood cultures to rule out infection, a comprehensive metabolic panel (CMP) to assess for electrolyte imbalances, hypoglycemia, and liver or kidney damage as evidenced by blood urea nitrogen (BUN), creatinine, and liver enzyme levels. Additional laboratory testing consists of an ammonia test, a lactate level, and a disseminated intravascular coagulation (DIC) panel [2].

Imaging studies include magnetic resonance imaging (MRI) and electroencephalogram (EEG). An MRI between 24 and 96 hours after the initial injury provides more information regarding the timing of the event, whereas an MRI between 96-120 hours after the injury provides information about the infant's prognosis [2]. The feasibility of an MRI shortly after delivery is dependent upon the patient's status. If the patient remains too unstable for transport to radiology, then an MRI is often delayed until the patient is stable enough to withstand transport [4].

Neonatal seizures often accompany HIE, thereby placing the patient at an increased risk of cognitive impairment, physical disability, epilepsy, and death [4]. Therefore, prompt seizure identification and treatment are two important factors in preventing further damage to the developing brain. According to researchers, seizure activity is confirmed when "a sudden change in the heart rate or blood pressure occurs in addition to the involvement of clinical features, such as apnea, eye deviation, focal tonic stiffening, or pupillary dilatation" ([4] pp. 25-26). Neonatal seizures often go unnoticed because of their subtle nature, which makes it challenging to capture seizure activity. However, continuous EEG monitoring combined with video recordings allow clinicians to more accurately assess seizure activity [4].

\section{Etiology and Pathophysiology}

Brain injury associated with HIE is a multifactorial process comprised of three phases: primary energy failure, latent phase, and secondary energy failure [1].

\section{Energy failure}

Primary energy failure occurs during the initial injury. Reduced brain tissue perfusion impairs oxygen and glucose uptake, which impairs adenosine triphosphate (ATP) production and increases lactic acid production due to anaerobic metabolism [1]. A decreased concentration of ATP causes failure of the cellular sodium and potassium pumps, which increases glutamate production and secretion. Glutamate permits intracellular movement of calcium and sodium. The influx of sodium into cells causes cellular swelling and subsequent rupture, which is followed by cell death [1]. This causes "cerebral edema, ischemia, and microvascular damage with resultant necrosis and/or apoptosis". In severe cases of HIE, the ruptured cell contents cause an exaggerated inflammatory response that can result in damage to the white matter of the brain, leading to scar tissue formation [1]. However, in less severe cases of HIE, the cells may either recover normal function or induce apoptosis, which stimulates programmed cell death while maintaining cellular membranes. This prevents further damage associated with the inflammatory response seen in severe HIE [1].

\section{Latent phase}

The latent phase is characterized by restoration of cerebral blood flow during a brief recovery. The duration of the latent phase is associated with the severity of the initial injury. For example, whereas mild HIE may present with a longer latent phase, a moderate to severe injury typically presents with a shorter latent phase [1]. Although it is difficult to determine the timing of each of the individual phases, the latent phase is considered the best time to initiate body cooling in neonates who meet the specific criteria [1].

\section{Secondary energy failure}

Secondary energy failure is also referred to as a reperfusion injury. This phase typically occurs six hours after the initial perinatal event, which indicates that therapeutic intervention should be implemented within six hours of the injury [1]. Reperfusion injury is characterized by "oxidative stress, excitotoxicity, and inflammation" ([1], p., 4). Oxidative stress is extremely deleterious to the neonatal brain. During the initial hypoxic ischemic injury, iron that was once bound to protein becomes free iron, which leads to free radical production [1]. Neonates have limited antioxidant stores; therefore, they have a reduced ability to eliminate free radicals. This places neonates at a greater risk for oxidative stress. The inverse relationship between free radical production and antioxidant elimination increases the likelihood of cellular membrane damage, cell necrosis, or apoptosis, which are the hallmarks of oxidative stress [1].

Excitotoxicity occurs secondary to extreme concentrations of extracellular neurotransmitters, such as glutamate 
that binds and activates excitatory receptors. Glutamate is essential for several pathways such as "hearing, somatosensory function, learning, and memory ([1], p.4). Excessive activation of excitatory receptors causes an influx of sodium and calcium into neural cells, causing potentially irreversible damage. These events are presumed to be the cause of long term sequelae of HIE that interrupt normal growth and development [1].

\section{Treatment: Therapeutic Hypothermia}

An estimated $62 \%$ of neonates who present with a hypoxic brain injury will die or develop moderate to severe neurocognitive deficits without treatment; however, that statistic drops to $41 \%$ with treatment [5]. After completing a meta-analysis that consisted of approximately 11,000 studies and a total of 3,592 patients, researchers concluded that the mortality risk associated with HIE decreased by $26 \%$ compared to those neonates who did not receive therapeutic hypothermia [5]. These statistics support the implementation of therapeutic hypothermia as the gold standard of HIE treatment in patients who meet the criteria. The initial perinatal hypoxic event causes the temperature of the brain to increase, which increases metabolic demands and induces production of inflammatory mediators that are toxic to brain structures [5]. Therapeutic hypothermia, an FDA approved treatment for HIE in neonates, aims to decrease the metabolic demands of the brain by lowering the body's core temperature. The goal of hypothermia is to decrease the metabolic demands of the body post injury, which allows cells to recover and ultimately decreases the severity of permanent brain injury [5]. Research indicates that lowering the body temperature by one degree Celsius results in a six to ten percent decrease in the body's metabolic demands; however, neuroprotection in newborns is seen when the brain temperature decreases by two to five degrees Celsius [5]. Clinical trials suggest that neurons and oligodendrocytes have the best chance of survival when hypothermia is initiated within the latent phase (90 minutes to three hours after the initial insult). In fact, when initiated within three hours, therapeutic hypothermia restores brain function to near baseline levels [5]. In contrast, the rate of neuronal and oligodendrocyte survival dramatically decreases when hypothermia is initiated at the end of the latent phase between five to six hours after the injury. Hypothermia is no longer effective if therapy is initiated during the secondary energy failure stage. Therefore, it is imperative that therapeutic hypothermia be implemented within six hours of injury; however, optimal outcomes are noted when intervention is initiated within three hours [5].

There are two methods of therapeutic hypothermia: selective head cooling and whole body cooling. The same meta-analysis also concluded that there was no difference in the mortality rate when selective head cooling was compared to total body cooling [5]. Although researchers have not seen a difference in outcomes, the medical community recommends whole body cooling over selective head cooling for several reasons [5]. For example, whole body cooling is easier for clinical application because it is not typically associated with hyperthermic temperature fluctuations during the rewarming phase such as with selective head cooling. In addition, whole body cooling cools all parts of the brain, whereas selective head cooling only cools the cortical portion of the brain. Therefore, whole body cooling is both more applicable to practice, and it more effectively cools the brain [5].

Criteria for therapeutic hypothermia include greater than or equal to 36 weeks' gestation at birth, at least 1,800 grams at delivery, less than 6 hours of age, an arterial pH less than or equal to seven, and a base deficit greater than or equal to negative $16 \mathrm{mEq} / \mathrm{L}$ [5]. In addition, one of the following must be present: History of any acute perinatal event in combination with an Apgar score less than or equal to five at 10 minutes of life or continued need for positive pressure ventilation or intubation within 10 minutes of delivery [5]. All neonates must demonstrate signs of moderate to severe encephalopathy within six hours of delivery. Exclusion criteria include less than 36 weeks' gestation, more than six hours old, $\mathrm{pH}$ greater than 7.15 , base deficit less than negative 10 $\mathrm{mEq} / \mathrm{L}$, chromosomal anomalies, and birth weight less than 1,800 grams [5].

Current guidelines suggest initiating therapeutic hypothermia as soon as possible for patients who meet inclusion criteria. The ideal core body temperature ranges from 33 to 34 degrees Celsius with a goal of 33.5 degrees Celsius. It is important to carefully monitor the patient's temperature with an esophageal temperature probe to ensure that the baby's temperature does not fall below 33 degrees Celsius [5]. Once the patient has reached the goal temperature, continue hypothermia for 72 hours. After this time period has ended, guidelines recommend that medical personnel slowly rewarm the neonate to a normal temperature over the course of six to nine hours to avoid seizure activity, hypotension, and vasodilation related to rapid rewarming. This equates to a rewarming rate of 0.5 degrees Celsius every hour for six hours [5].

Therapeutic hypothermia is a reputable intervention for HIE; however, continued research is necessary to further enhance our understanding of the disease process and treatment. In addition, follow up will be an important component of ongoing care, but education on this topic can wait until discharge when medical professionals can more accurately predict the patient's outcome [5].

\section{Side Effects Associated with Therapeutic Hypothermia}

\section{Pharmacokinetic changes}

It is imperative that the NNP understands how hypothermia affects pharmacokinetics, which concerns drug absorption, distribution, metabolism, and excretion. Changes in pharmacokinetics necessitate consideration of drug dosage, interval, and administration to avoid toxicity. Hypothermia changes the route of blood flow. For example, hypothermia decreases cardiac output and shunts blood to vital organs such as the brain and heart [6]. Drug metabolism and elimination are reduced as blood preferentially shunts away from the extremities, liver, and kidneys, which decrease the volume of distribution [6]. This ultimately places the 
neonate at an increased risk of drug toxicity; therefore, the NNP must be aware of the pharmacokinetic changes that occur due to therapeutic hypothermia [6].

Rewarming the body causes drugs that had been previously stored in the peripheral tissues to recirculate, which potentially exposes the neonate to dangerously high drug concentrations. This places the neonate at an even greater risk of toxicity [6]. The NNP must be conscientious in order to avoid adverse effects associated with drug toxicity; therefore, it is important to periodically follow drug levels of toxic drugs during this time.

\section{Response to insulin}

Therapeutic hypothermia decreases the body's response to insulin; therefore, neonates are at an increased risk of developing hyperglycemia [7]. Hyperglycemia in conjunction with HIE significantly increases the mortality risk. Careful blood glucose monitoring is necessary, and a continuous insulin infusion during therapy may be warranted if the patient experiences sustained hyperglycemia [7]. Neonates consequently experience increased responsiveness to insulin during the rewarming phase of therapeutic hypothermia, which predisposes them to hypoglycemia especially if he/she receives a continuous insulin infusion during this time. This increased risk of hypoglycemia warrants further glycemic surveillance until rewarming is complete [7].

\section{Implications for the NNP}

The advanced practice registered nurse (APRN) should have a basic understanding of HIE, its clinical presentation, diagnostic tools, and available treatment options to provide quality nursing care. Familiarity with HIE is crucial to early diagnosis and treatment; therefore, the patient's care team consists of multiple disciplines. This multidisciplinary team approach provides the patient with the best treatment and outcome. The nurse practitioner should know the predisposing maternal and fetal factors that increase the likelihood of an HIE diagnosis to create a differential diagnosis based on both pertinent positive and negative clinical findings.

The NNP must be aware of the clinical manifestations associated with HIE, but exclude all other possible explanations before arriving at a definitive diagnosis. For example, the NNP must be aware that sepsis, inborn errors of metabolism, neonatal stroke, genetic anomalies, and hemorrhage have similar manifestations [2]. Therefore, it is essential that the APRN utilizes the appropriate diagnostic measures when evaluating a neonate with a predisposition for HIE to avoid an incorrect diagnosis. With the help of the comprehensive medical team and current diagnostic tools, the APRN can make a conclusive diagnosis once all other possibilities have been excluded. The NNP should also be cognizant of potential adverse effects of hypothermia, which include prolonged medication half-lives, inhibition of antimicrobial activity, increased demand for oxygen, transient hyperglycemia, and prolongation of the QT interval [7].

The NNP must also be able to navigate the social aspect of an HIE diagnosis. It is important to provide accurate parent updates without use of medical jargon, so that they can better understand this diagnosis. The NNP can encourage parents to participate in treatment plans because parents are often the patient's most important advocates. Parent/ caregiver involvement should not be understated. Discussion of outcomes is also important. Provide parents with honest prognoses while simultaneously showing support and understanding, so that they can understand the gravity of an HIE diagnosis. The NNP should also prepare parents upon discharge for the developmental follow up appointments. The developmental team will ensure the best possible neurological and developmental outcomes depending on the degree of brain damage. It is important for the NNP to know the long-term outlook and treatment options that this literature review discusses in order to guide parents through this unknown territory. Therefore, the NNP must be up to date on current diagnostic and treatment options in order to provide the best nursing care possible.

\section{References}

1. Allen K, Brandon D (2011) Hypoxic ischemic encephalopathy: pathophysiology and experimental treatments. Newborn Inf Nurs Rev 11: 125-133.

2. Sinha R, Venkatnarayan K, Negi V, et al. (2018) The effect of whole body cooling in asphyxiated neonates with resource limitation: Challenges and experience. J Clin Neonatol 7: 7-11.

3. El-Gamasy M, Alarabawy R (2018) Relation of serum creatinine to Sarnat scoring and brain computerized tomography of neonates with hypoxic ischemic encephalopathy. A single-center experience. J Pediatr Neurosci 13: 437-442.

4. Lin YK, Hwang-Bo S, Seo Ym, et al. (2021) Clinical seizures and unfavorable brain MRI patterns in neonates with hypoxic ischemic encephalopathy. Medicine 100: e25118.

5. Abate BB, Bimerew M, Gebremichael B, et al. (2021) Effects of therapeutic hypothermia on death among asphyxiated neonates with hypoxic-ischemic encephalopathy: a systematic review and meta-analysis of randomized control trials. PLoS One 16: 1-20.

6. Holmes A (2017) NICU primer for pharmacists. ( $1^{\text {st }}$ edn) American Society of Health-System Pharmacists, Maryland, USA.

7. Rao R, Trivedi S, Vesoulis Z, et al. (2017) Safety and shorter-term outcome of therapeutic hypothermia in preterm neonates 34-35 weeks gestational age with hypoxic-ischemic encephalopathy. J Pediatr 183: 37-42.

DOI: $10.36959 / 395 / 517$

Copyright: (C) 2022 Gill JA. This is an open-access article distributed under the terms of the Creative Commons Attribution License, which permits unrestricted use, distribution, and reproduction in any medium, provided the original author and source are credited. 